\title{
First metatarsophalangeal joint range of motion is associated with lower limb kinematics in individuals with first metatarsophalangeal joint osteoarthritis
}

\author{
Jamie J. Allan ${ }^{1,2}$, Jodie A. McClelland ${ }^{1,2,3}$, Shannon E. Munteanu ${ }^{1,2}$, Andrew K. Buldt ${ }^{1,2}$, Karl B. Landorf ${ }^{1,2}$,
} Edward Roddy ${ }^{4,5}$, Maria Auhl ${ }^{1}$ and Hylton B. Menz ${ }^{1,2^{*}}$ (i)

\begin{abstract}
Background: Osteoarthritis of the first metatarsophalangeal joint (1st MTP joint OA) is a common and disabling condition that results in pain and limited joint range of motion. There is inconsistent evidence regarding the relationship between clinical measurement of 1st MTP joint maximum dorsiflexion and dynamic function of the joint during level walking. Therefore, the aim of this study was to examine the association between passive nonweightbearing (NWB) 1st MTP joint maximum dorsiflexion and sagittal plane kinematics in individuals with radiographically confirmed 1 st MTP joint OA.
\end{abstract}

Methods: Forty-eight individuals with radiographically confirmed 1st MTP joint OA (24 males and 24 females; mean age 57.8 years, standard deviation 10.5) underwent clinical measurement of passive NWB 1st MTP joint maximum dorsiflexion and gait analysis during level walking using a 10-camera infrared Vicon motion analysis system. Sagittal plane kinematics of the 1st MTP, ankle, knee, and hip joints were calculated. Associations between passive NWB 1st MTP joint maximum dorsiflexion and kinematic variables were explored using Pearson's $r$ correlation coefficients.

Results: Passive NWB 1st MTP joint maximum dorsiflexion was significantly associated with maximum 1st MTPJ dorsiflexion ( $r=0.486, p<0.001)$, ankle joint maximum plantarflexion $(r=0.383, p=0.007)$, and ankle joint excursion $(r=0.399, p=0.005)$ during gait. There were no significant associations between passive NWB 1 st MTP joint maximum dorsiflexion and sagittal plane kinematics of the knee or hip joints.

Conclusions: These findings suggest that clinical measurement of 1st MTP joint maximum dorsiflexion provides useful insights into the dynamic function of the foot and ankle during the propulsive phase of gait in this population.

\footnotetext{
* Correspondence: h.menz@latrobe.edu.au

${ }^{1}$ Discipline of Podiatry, School of Allied Health, Human Services and Sport, La

Trobe University, Melbourne, Victoria 3086, Australia

${ }^{2}$ La Trobe Sport and Exercise Medicine Research Centre, School of Allied

Health, Human Services and Sport, La Trobe University, Melbourne, Victoria

3086, Australia

Full list of author information is available at the end of the article
}

(C) The Author(s). 2020 Open Access This article is licensed under a Creative Commons Attribution 4.0 International License, which permits use, sharing, adaptation, distribution and reproduction in any medium or format, as long as you give appropriate credit to the original author(s) and the source, provide a link to the Creative Commons licence, and indicate if changes were made. The images or other third party material in this article are included in the article's Creative Commons licence, unless indicated otherwise in a credit line to the material. If material is not included in the article's Creative Commons licence and your intended use is not permitted by statutory regulation or exceeds the permitted use, you will need to obtain permission directly from the copyright holder. To view a copy of this licence, visit http://creativecommons.org/licenses/by/4.0/ The Creative Commons Public Domain Dedication waiver (http://creativecommons.org/publicdomain/zero/1.0/) applies to the data made available in this article, unless otherwise stated in a credit line to the data. 


\section{Background}

Osteoarthritis of the first metatarsophalangeal joint (1st MTP joint OA) has been recognised as one of the most common causes of foot pain in middle-aged and older people [1]. The condition affects $8 \%$ of individuals aged over 50 years and leads to disability, poorer healthrelated quality of life, and impaired locomotor function [1]. 1st MTP joint OA is characterised by joint pain and stiffness, dorsal exostosis formation, and reduced 1st MTP joint dorsiflexion range of motion [2]. The presence of adequate 1st MTP joint dorsiflexion is essential during the terminal stance and pre-swing phases of gait to enable smooth forward progression of the body over the foot [3]. As a consequence of limited motion within the joint, individuals with 1st MTP joint OA adopt an altered gait pattern, characterised by reduced step length and shorter stance duration $[4,5]$.

Three studies have explored the relationship between clinical measurement of 1st MTP joint motion and dynamic function during walking, with inconsistent findings $[3,6,7]$. In pain-free, healthy individuals, Nawoczenski et al. [3] found significant associations between 1st MTP joint maximum dorsiflexion during walking and active weightbearing (Pearson's $r=0.80$ ), passive weightbearing $(r=0.61)$ and passive nonweightbearing $(r=0.67)$ 1st MTP joint ROM. Similarly, in asymptomatic individuals, Jarvis et al. [6] found a significant association $(r=0.32)$ between 1st MTP joint maximum dorsiflexion and maximal dorsiflexion during walking. In contrast, Halstead et al. [7] found no significant association between passive 1st MTP joint maximum dorsiflexion and 1st MTP joint maximum dorsiflexion during walking in individuals with limited 1st MTP joint motion (as determined by Jack's test [8] in relaxed standing). To the best of our knowledge, no studies have examined this association in individuals with radiographically-confirmed 1st MTP joint OA.

Therefore, the primary aim of this study was to determine whether there is an association between passive non-weightbearing (NWB) 1st MTP joint maximum dorsiflexion and sagittal plane kinematics in individuals with radiographically confirmed 1st MTP joint OA. Doing so will provide insight into the underlying mechanisms responsible for gait alterations in individuals with this condition.

\section{Methods}

\section{Participants}

Participants for this study were drawn from a larger randomised trial evaluating the effectiveness of shoestiffening inserts for 1st MTP joint OA, the details of which have been published previously [9]. The La Trobe University Human Ethics Committee provided ethical approval (number HEC15-128) and all participants provided written informed consent prior to enrolment. Briefly, individuals with 1st MTP joint OA were recruited by advertisements placed in local newspapers, posters placed in senior citizens' centres and retirement villages, mail-out advertisements to health-care practitioners in Melbourne, mail-outs to people currently accessing podiatry services at the La Trobe University Health Sciences Clinic, and through social networking media (e.g. Facebook, Twitter). Inclusion criteria were: (i) 18 years of age or older, (ii) pain in the 1st MTP joint on most days for at least 12 weeks, (iii) pain rated at least $30 \mathrm{~mm}$ on a $100 \mathrm{~mm}$ visual analogue scale (VAS), (iv) pain upon palpation of the dorsal aspect of the 1st MTP joint, (v) able to walk household distances (> $50 \mathrm{~m}$ ) without the aid of a walker, crutches or cane, and (vi) willing to have their foot $\mathrm{x}$-rayed. Exclusion criteria included: (i) previous first MTP joint surgery, (ii) currently pregnant, (iii) significant first MTP joint deformity including hallux valgus, (iv) presence of any systemic inflammatory condition such as gout or rheumatoid arthritis, (v) an inability to speak and read English, and (vi) cognitive impairment.

\section{Clinical and radiographic assessment}

Participant characteristics (such as age, sex, weight, height, education and income level), major medical conditions and number of medications were obtained via a structured questionnaire. Height and weight were measured using a stadiometer and digital scales, and body mass index (BMI) was calculated as weight $(\mathrm{kg})$ / height $(\mathrm{m})^{2}$. Static foot posture was assessed using the Foot Posture Index [10]. Passive NWB 1st MTP joint maximum dorsiflexion as measured using a reliable goniometric technique [11]. The first metatarsal and proximal phalanx of the hallux were bisected in the sagittal plane. A dorsiflexion force was applied to the hallux until end range of motion was reached, allowing the first ray to maximally plantarflex. The angle between the two lines was then measured via a handheld goniometer (see Fig. 1). The reliability of this test has been shown to be excellent in healthy individuals [12] and individuals with 1st MTP joint OA [11] (intra-class correlation coefficient $=0.95)$. Clinical features associated with 1st MTP joint OA (pain on palpation, dorsal exostosis, joint effusion, pain on motion, hard-end feel and crepitus) were documented [11]. The presence of radiographic 1st MTP joint OA was determined at baseline using the La Trobe University radiographic atlas [13]. The atlas incorporates weightbearing dorso-plantar and lateral radiographs to document the presence of OA based on observations of osteophytes and joint space narrowing. Osteophytes were recorded as absent (score $=0$ ), small $($ score $=1)$, moderate $($ score $=2)$ or severe $($ score $=3)$. Joint space narrowing was recorded as none (score $=0$ ), 


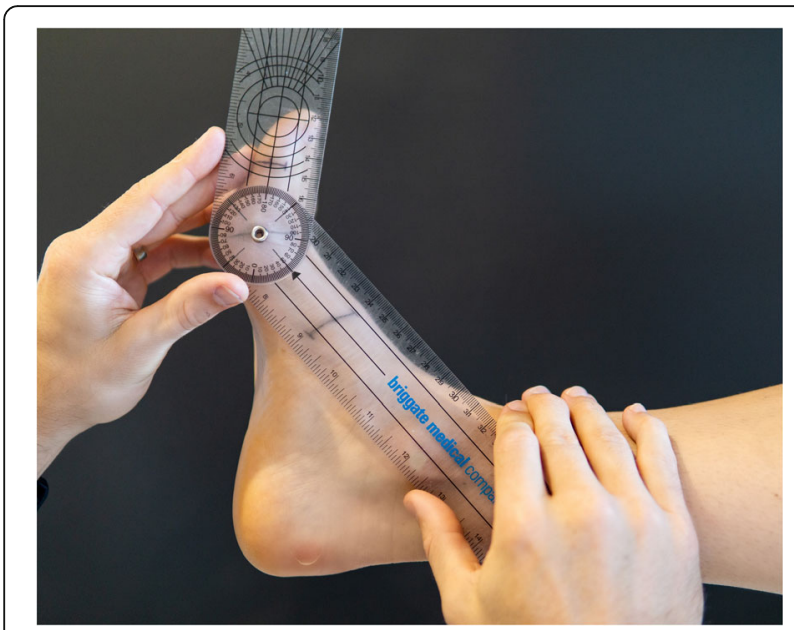

Fig. 1 Measurement of passive NWB 1st MTP joint maximum dorsiflexion

definite $($ score $=1)$, severe $($ score $=2)$ or joint fusion $($ score $=3)$. Radiographic OA using this atlas is defined as a score of 2 or more for osteophytes or joint space narrowing on either dorso-plantar and lateral views. The atlas has been shown to have good to excellent intraand inter-rater reliability for grading 1 st MTP joint OA ( $\mathrm{k}$ range 0.64 to 0.95 ) [13].

\section{Biomechanical assessment}

Biomechanical assessment was performed to evaluate sagittal plane kinematics of the 1st MTP, hip, knee and ankle joints. Kinematics were measured using a 10camera infrared motion analysis system (Vicon Motion Systems Ltd., UK). Dorsiflexion of the hallux during walking was measured by attaching six passive retroreflective markers to the medial forefoot (3 markers) and proximal phalanx of the hallux (3 markers) as required for calculation of 1st MTP joint kinematics using a modification of the Salford Foot Model [14]. This model has been used to assess 1st MTP joint kinematics with acceptable reliability [14]. In addition, 32 markers were fixed to anatomical landmarks of the trunk, pelvis and lower limb based on the modified Helen Hayes marker set $[15,16]$, as well as a customised model to allow for segmental definition and functional joint calibration. Marker trajectories were collected at a frequency of 100 $\mathrm{Hz}$, and all lower limb joint kinematics were calculated based on Euler angles and described in terms of movement of the distal segment relative to the proximal segment. Data were collected and averaged from the middle stride of six 10-m walking trials for each condition at self-selected walking speed. Participants were equipped with 'gait shoes' with a laced fastening and canvas upper, customised with cut-outs in order to allow clear visualisation of the foot markers (Fig. 2). The minimum and

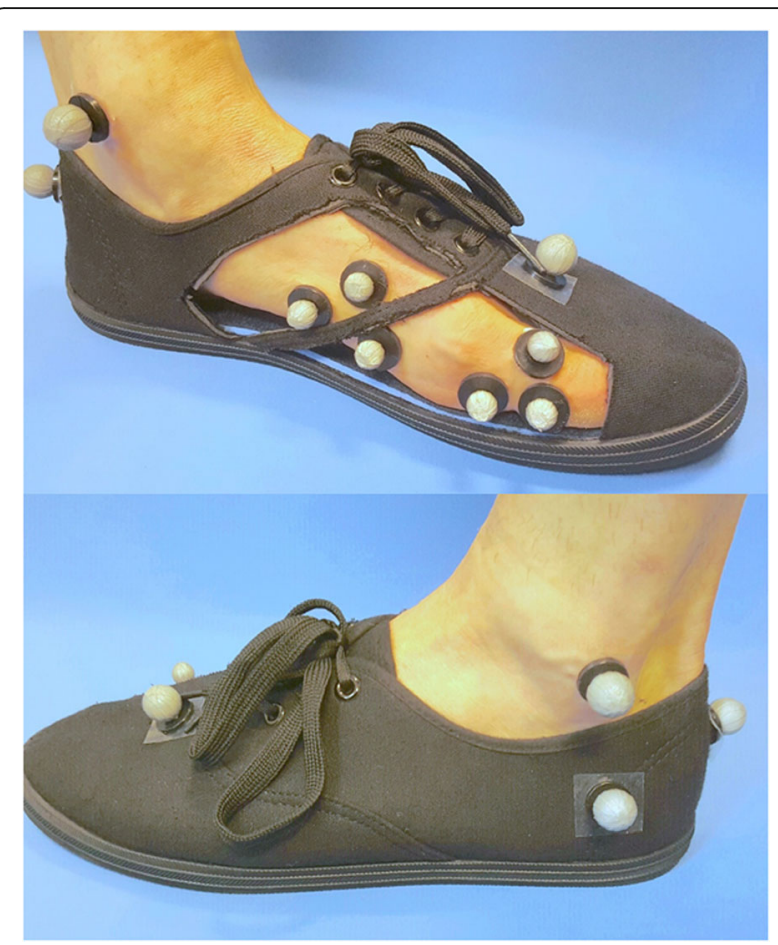

Fig. 2 Location of foot markers used for kinematic analysis. Figure from Munteanu SE, Landorf KB, McClelland JA, Roddy E, Cicuttini FM, Shiell A, Auhl M, Allan JJ, Buldt AK, Menz HB: Shoe-stiffening inserts for first metatarsophalangeal joint osteoarthritis (the SIMPLE trial): study protocol for a randomised controlled trial. Trials 2017, 18:198

maximum angles throughout the stance phase of gait were extracted from each of the six strides and averaged to represent gait for each individual. The range of motion of each joint was calculated by subtracting the minimum angle from the maximum angle.

\section{Statistical analysis}

Statistical analysis was undertaken using SPSS version 26.0 (IBM Corp, NY, USA). We included one foot only for each participant. The symptomatic side was included (either right or left), and in the case of bilateral symptoms, the most symptomatic foot only was analysed. All data were screened for normality and outliers. Analysis was then undertaken in three stages. Firstly, associations between passive NWB 1st MTP joint maximum dorsiflexion, participant characteristics (age, height, weight, BMI and pain severity), temporo-spatial gait characteristics (velocity, cadence and step length) and the kinematic gait variables were analysed using Pearson's $r$ correlation coefficients, as these variables were considered to be possible confounders. Secondly, associations between passive NWB 1st MTP joint maximum dorsiflexion and the kinematic gait variables were analysed, and where necessary, adjusted for confounders using partial Pearson's $r$ correlation coefficients. Statistical 
significance was set at $p<0.05$. Correlation coefficients were interpreted using the following cut-off values: 0 to 0.29 (weak), 0.30 to 0.49 (moderate), 0.50 to 1.00 (large) [17]. Finally, for all significant correlations, $r^{2}$ values were calculated to express the proportion of variance in kinematic variables explained by 1st MTP joint maximum dorsiflexion. Sagittal plane kinematic variables that were included in the analysis were: 1st MTP joint maximum dorsiflexion, ankle joint maximum plantarflexion, ankle joint maximum dorsiflexion, ankle joint excursion, knee joint maximum extension, knee joint maximum flexion, knee joint excursion, hip joint maximum extension, hip joint maximum flexion and hip joint excursion.

\section{Results}

\section{Participants}

One hundred participants (45 men and 55 women, age 24 to 82 years, mean 57.5 [SD 10.3]) were recruited for the randomised trial. Of these, 54 participants were available and consented to biomechanical analysis, and complete data were available for 48 participants (24 males and 24 females). Characteristics of these participants are reported in Table 1.

Table 1 Participant characteristics. Values are mean (SD) unless otherwise noted

\begin{tabular}{ll}
\hline Demographics and anthropometrics & \\
\hline Age - years & $57.8(10.5)$ \\
Female $-\mathrm{n}(\%)$ & $24(50)$ \\
Height $-\mathrm{cm}$ & $168.1(8.3)$ \\
Weight $-\mathrm{kg}$ & $80.0(14.2)$ \\
Body mass index $-\mathrm{kg} / \mathrm{m}^{2}$ & $28.4(4.6)$ \\
Clinical features & \\
Passive NWB 1st MTP joint maximum dorsiflexion - & $45.1(10.7)[17-$ \\
mean (SD) [range], degrees & \\
Pain duration - median [range], months & $62]$ \\
Pain on palpation - $\mathrm{n}(\%)$ & $48.0[6-432]$ \\
Palpable dorsal exostosis - $\mathrm{n}(\%)$ & $48(100)$ \\
Pain on motion of 1st MTP joint - $\mathrm{n}(\%)$ & $48(100)$ \\
Hard-end feel when dorsiflexed - $\mathrm{n}(\%)$ & $35(72.9)$ \\
Crepitus - $\mathrm{n}$ (\%) & $44(91.7)$ \\
Radiographic features - $\mathrm{n}(\%)^{\mathrm{b}}$ & $16(33.3)$ \\
Dorsal osteophytes & \\
Dorsal joint space narrowing & $44(91.7)$ \\
Lateral osteophytes & $43(89.6)$ \\
Lateral joint space narrowing & $44(91.7)$ \\
Radiographic 1st MTP joint OA & $44(91.7)$ \\
\hline
\end{tabular}

adata non-normally distributed

$\mathrm{b}_{\text {score }}>0$ using La Trobe Radiographic Atlas [13]

cat least one score of 2 for osteophytes or joint space narrowing from either view, using case definition from La Trobe Radiographic Atlas [13]
Sagittal plane kinematics of the 1st MTP, ankle, knee and hip joints

Sagittal plane kinematics of the 1st MTP, ankle, knee and hip joints in individuals with 1st MTP joint OA are reported in Table 2 and visually presented in Fig. 3. Mean dynamic 1st MTP joint maximum dorsiflexion was 25.4 (SD 6.7) degrees.

\section{Associations between passive NWB 1st MTP joint maximum dorsiflexion and kinematic variables}

There were no significant associations between passive NWB 1st MTP joint maximum dorsiflexion and participant characteristics (age, height, weight, BMI) or temporo-spatial gait characteristics (velocity, cadence and step length), so no adjustment for confounding was required. Associations between passive NWB 1st MTP joint maximum dorsiflexion and sagittal plane kinematics are shown in Table 3. Passive NWB 1st MTP joint maximum dorsiflexion was moderately associated with dynamic 1st MTP joint maximum dorsiflexion $\left(r=0.486, p<0.01 ; r^{2}=0.236\right)$, ankle joint maximum plantarflexion $\left.r=0.383, p<0.01 ; r^{2}=0.147\right)$, and ankle joint excursion $\left(r=0.399, p<0.01 ; r^{2}=0.159\right)$. A scatterplot of the association between passive NWB 1st MTP joint maximum dorsiflexion and 1st MTP joint maximum dorsiflexion during stance phase is shown in Fig. 4.

\section{Discussion}

This study examined the relationship between passive NWB 1st MTP joint maximum dorsiflexion and sagittal plane kinematics in individuals with 1st MTP joint OA. Our findings indicate that individuals with less passive NWB 1st MTPJ maximum dorsiflexion exhibit less 1st MTP joint maximum dorsiflexion, less ankle joint maximum plantarflexion and ankle joint excursion during level walking. The magnitude of these associations was

Table 2 Descriptive statistics for sagittal plane kinematics (stance phase) in individuals with 1st MTP joint OA. Values are degrees

\begin{tabular}{lll}
\hline Kinematic variable & Mean (SD) & Range \\
\hline 1st MTP joint - maximum dorsiflexion & $25.4(6.7)$ & $13.8-39.5$ \\
Ankle joint - maximum plantarflexion & $7.3(5.4)$ & $-3.0--21.6$ \\
Ankle joint - maximum dorsiflexion & $15.6(3.5)$ & $9.5-25.3$ \\
Ankle joint - total excursion & $22.8(4.4)$ & $13.9-32.9$ \\
Knee joint - maximum extension & $0.7(5.5)$ & $-11.7--11.4$ \\
Knee joint - maximum flexion & $35.6(6.5)$ & $18.5-51.3$ \\
Knee joint - total excursion & $36.3(6.4)$ & $16.1-51.8$ \\
Hip joint - maximum extension & $13.4(7.3)$ & $1.6-27.2$ \\
Hip joint - maximum flexion & $34.2(7.1)$ & $19.8-48.4$ \\
Hip joint - total excursion & $47.5(5.2)$ & $35.1-57.0$ \\
\hline
\end{tabular}



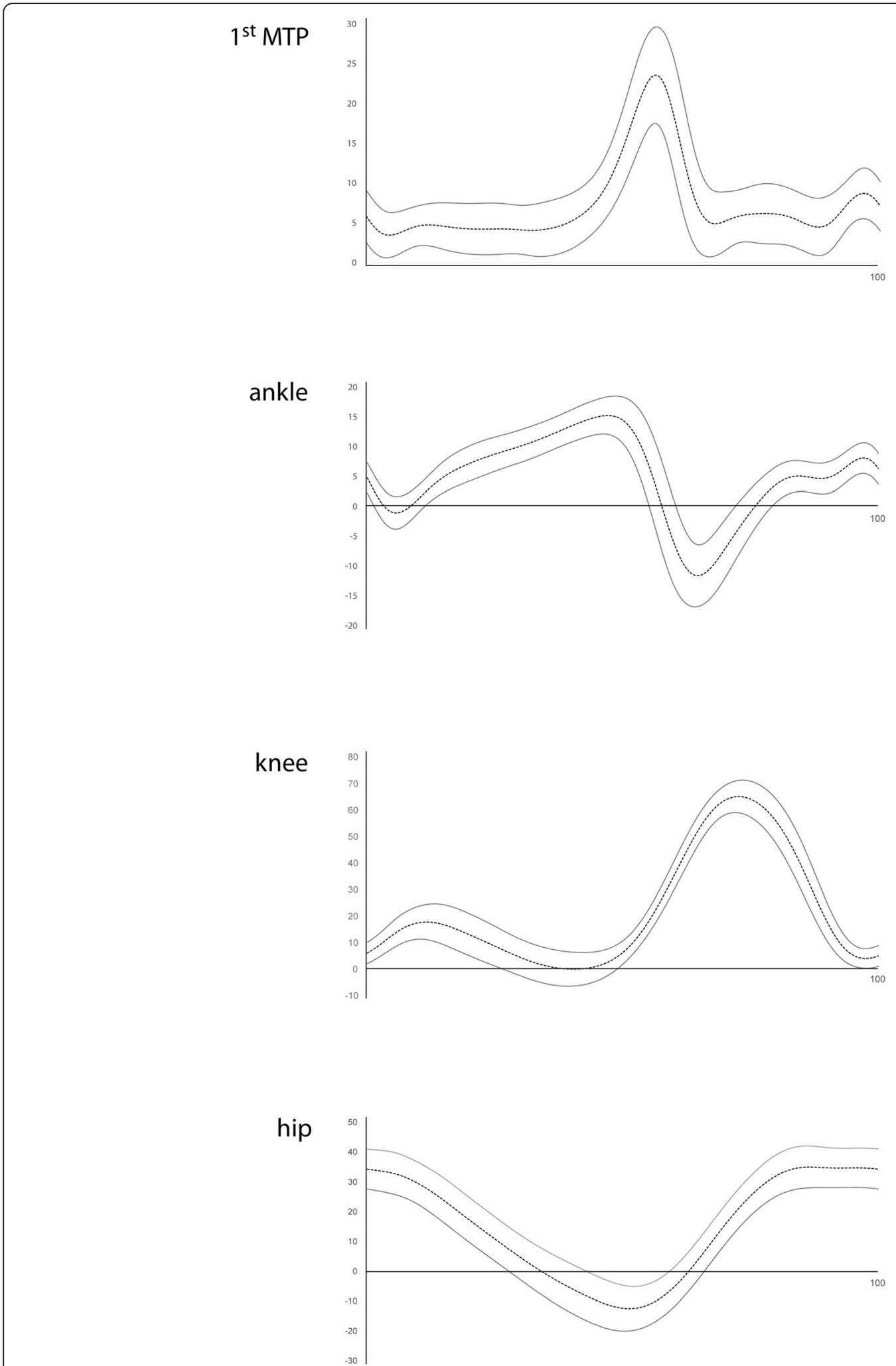

Fig. 3 Sagittal plane kinematics (mean \pm standard error, degrees) of the 1st MTP, ankle, knee and hip joints during level walking in individuals with 1st MTP joint OA. X-axis represents percentage of the gait cycle 
Table 3 Associations between passive NWB 1st MTP joint maximum dorsiflexion and lower limb kinematics. Values are Pearson's $r$ correlation coefficients and $p$-values

\begin{tabular}{lll}
\hline Kinematic variable & $r$ & $p$ \\
\hline 1st MTP joint - maximum dorsiflexion & 0.486 & $<0.001^{*}$ \\
Ankle joint - maximum plantarflexion & 0.383 & $0.007^{*}$ \\
Ankle joint - maximum dorsiflexion & -0.068 & 0.646 \\
Ankle joint - excursion & 0.399 & $0.005^{*}$ \\
Knee joint - maximum extension & 0.150 & 0.310 \\
Knee joint - maximum flexion & -0.036 & 0.810 \\
Knee joint - excursion & 0.090 & 0.542 \\
Hip joint - maximum extension & 0.135 & 0.359 \\
Hip joint - maximum flexion & -0.191 & 0.193 \\
Hip joint - excursion & -0.067 & 0.652 \\
\hline
\end{tabular}

* significant at $p<0.05$

moderate, with $r^{2}$ values indicating that passive NWB 1st MTP joint maximum dorsiflexion can explain approximately 24,15 and $16 \%$ of 1 st MTP joint maximum dorsiflexion, ankle joint maximum plantarflexion and ankle joint excursion, respectively. These findings are consistent with previous studies that indicate the reduction in range of motion associated with $\mathrm{OA}$ impairs the normal propulsive function of the foot $[4,5]$.

Passive NWB 1st MTP joint maximum dorsiflexion in our sample ranged from 17 to 62 degrees, with a mean of 45 degrees. Using the same measurement technique in a population-based study of 517 people aged over 50 years with foot pain, Menz et al. [2] found that passive NWB 1st MTP joint maximum dorsiflexion was associated with the radiographic severity of $\mathrm{OA}$, with the most severe radiographic category demonstrating a mean value of 42 degrees. This similarity suggests that our participants were towards the more severe end of the radiographic spectrum, which would be expected given that their reported duration of OA symptoms was 4 years. 1st MTP joint maximum dorsiflexion during gait in our study ranged from 14 to 40 degrees, with a mean of 25 degrees. Despite using different kinematic models, this is similar to the mean value reported by Canesco et al. (approximately 30 degrees) in 22 patients undergoing surgery for hallux rigidus [4].

The associations reported here are consistent with Nawoczenski et al. [3] and Jarvis et al. [6], who found significant correlations between passive NWB and dynamic 1st MTP joint dorsiflexion in a pain-free healthy populations ( $r=0.67$ and $r=0.32$, respectively). In contrast, Halstead et al. reported no significant association between passive and dynamic 1st MTP joint dorsiflexion $(r=0.186)$ [7]. However, in the Halstead et al. study, participants had limited passive 1st MTP joint maximum dorsiflexion in relaxed standing (positive Jack's test [8]), but normal ( $>50$ degrees) of passive NWB 1st MTP joint maximum dorsiflexion, indicative of "functional" hallux limitus. Our study, therefore, is the first to examine this association in individuals with symptomatic, radiographically-confirmed 1st MTP joint OA.

We found a significant positive association between passive NWB 1st MTP joint maximum dorsiflexion and ankle joint maximum plantarflexion during gait, which suggests that limited 1st MTP joint dorsiflexion may impair efficient propulsion. The presence of strategies to compensate for limited 1st MTP joint dorsiflexion has been reported in previous studies, where there was an increase in lateral forefoot loading and reduced ankle joint plantarflexion in the presence of 1st MTP joint OA $[5,18,19]$. These findings have previously been linked with the high- and low-gear push-off concept proposed

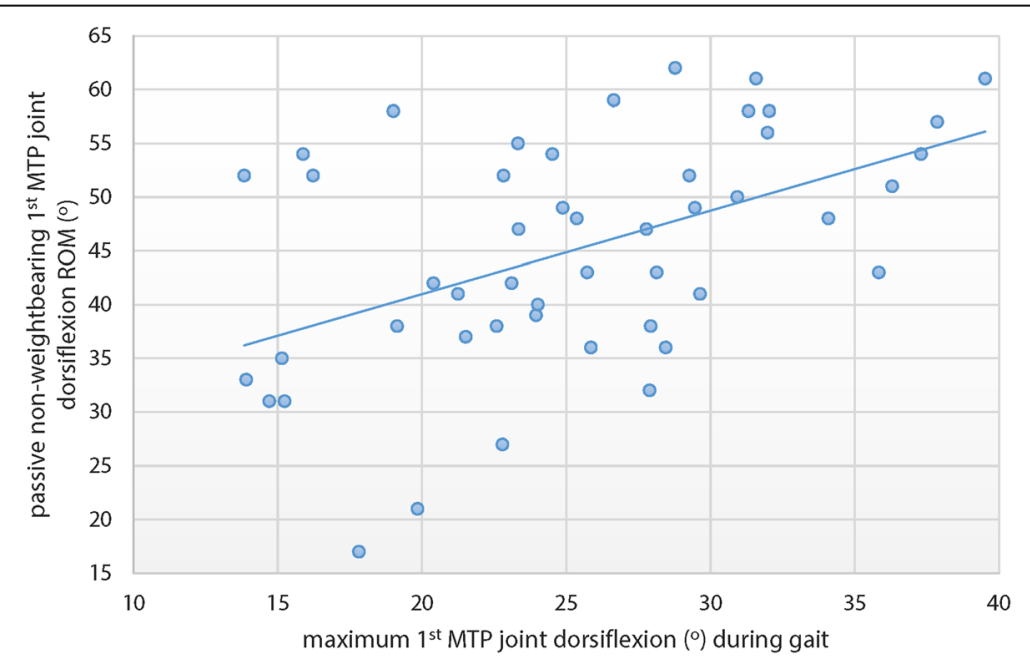

Fig. 4 Scatterplot of correlation between passive NWB 1st MTP joint maximum dorsiflexion and 1st MTP joint maximum dorsiflexion during the stance phase of gait in individuals with 1st MTP joint OA. Pearson's $r=0.486$ 
by Bojsen-Moller [20], whereby individuals with limited 1st MTP joint dorsiflexion fail to efficiently utilise the high-gear transverse axis (connecting the 1st and 2nd metatarsal heads) resulting in motion occurring through the low-gear oblique axis (connecting 2nd to 5th metatarsal heads) [5]. The low-gear propulsion causes a shorter lever arm between the ankle joint plantarflexors and forefoot, subsequently resulting in a higher lateral loading pattern and less efficient propulsion [5]. However, further kinematic and kinetic analyses are required to confirm this proposed mechanism.

This study has several methodological strengths, including radiographic confirmation of 1st MTP joint OA using a standardised atlas, a relatively large sample size for a kinematic study, and use of a reliable clinical measurement of passive NWB 1st MTP joint maximum dorsiflexion. However, the results of the study should be interpreted with respect to three key limitations. Firstly, due to the cross-sectional study design we cannot infer causality between passive NWB 1st MTP joint maximum dorsiflexion and kinematic changes. Secondly, kinetic data were not collected in this study, which would have allowed greater insight into the loading of the 1st MTP joint. Thirdly, our kinematic foot model was a simplified version of the Salford Foot Model [14], as participants needed to be tested while shod as part of the larger clinical trial. This precluded any analysis of the motion of the midfoot, which has been shown to be significantly altered in the presence of 1st MTP joint OA [4]. Finally, because participants were tested shod, we cannot exclude the influence of footwear on 1st MTP joint kinematics. However, the shoes used were of minimalist design with removal of large sections of the upper to accommodate the markers, so they were unlikely to have substantially influenced foot function.

In conclusion, this study identified that individuals with less passive NWB 1st MTP joint maximum dorsiflexion exhibit less dynamic 1st MTP joint maximum dorsiflexion, less ankle joint plantarflexion and less total ankle joint excursion during level walking. These findings suggest that clinical measurement of the 1st MTP joint provides useful insights into the dynamic function of the foot and ankle in this population. However, further study is required to determine the clinical importance of these observations.

\section{Acknowledgements}

HBM is currently a National Health and Medical Research Council Senior Research Fellow (ID: 1135995)

\section{Authors' contributions}

All authors were involved in drafting the article or revising it critically for important intellectual content, and all authors approved the final version to be submitted for publication. Study conception and design: SEM, HBM, JAM, KBL, ER. Acquisition of data: JJA, MA, AKB. Analysis and interpretation of data: $H B M, J J A$.

\section{Funding}

This study was funded by a project grant from the National Health and Medical Research Council of Australia (ID: 1105244).

\section{Availability of data and materials}

The datasets used and/or analysed during the current study are available from the corresponding author on reasonable request.

\section{Ethics approval and consent to participate}

Ethical approval was granted from the La Trobe University Human Ethics Committee (Reference 13-003), and written informed consent was obtained from all participants prior to the study.

\section{Competing interests}

None of the authors has a competing interest to declare.

\section{Author details}

'Discipline of Podiatry, School of Allied Health, Human Services and Sport, La Trobe University, Melbourne, Victoria 3086, Australia. ${ }^{2}$ La Trobe Sport and Exercise Medicine Research Centre, School of Allied Health, Human Services and Sport, La Trobe University, Melbourne, Victoria 3086, Australia. ${ }^{3}$ Discipline of Physiotherapy, School of Allied Health, Human Services and Sport, La Trobe University, Melbourne, Victoria 3086, Australia. ${ }^{4}$ Primary Care Centre Versus Arthritis, School of Primary, Community and Social Care, Keele University, Keele, Staffordshire ST5 5BG, UK. ${ }^{5}$ Haywood Academic Rheumatology Centre, Midlands Partnership NHS Foundation Trust, Haywood Hospital, Burslem, Staffordshire ST6 7AG, UK.

Received: 2 April 2020 Accepted: 26 May 2020

Published online: 08 June 2020

\section{References}

1. Roddy E, Thomas MJ, Marshall M, Rathod T, Myers H, Menz HB, Thomas E, Peat $\mathrm{G}$. The population prevalence of symptomatic radiographic foot osteoarthritis in community-dwelling older adults: the clinical assessment study of the foot. Ann Rheum Dis. 2015;74:156-63.

2. Menz HB, Roddy E, Marshall M, Thomas MJ, Rathod T, Myers H, Thomas E, Peat GM. Demographic and clinical factors associated with radiographic severity of first metatarsophalangeal joint osteoarthritis: cross-sectional findings from the clinical assessment study of the foot. Osteoarthritis Cartilage. 2015;23:77-82

3. Nawoczenski D, Baumhauer J, Umberger B. Relationship between clinical measurements and motion of the first metatarsophalangeal joint during gait. J Bone Joint Surg Am. 1999:81A:370-6.

4. Canseco K, Long J, Marks R, Khazzam M, Harris G. Quantitative characterization of gait kinematics in patients with hallux rigidus using the Milwaukee foot model. J Orthop Res. 2008;26:419-27.

5. Menz HB, Auhl M, Tan JM, Buldt AK, Munteanu SE. Centre of pressure characteristics during walking in individuals with and without first metatarsophalangeal joint osteoarthritis. Gait Posture. 2018:63:91-6.

6. Jarvis $\mathrm{HL}$, Nester CJ, Bowden PD, Jones RK. Challenging the foundations of the clinical model of foot function: further evidence that the root model assessments fail to appropriately classify foot function. J Foot Ankle Res. 2017;10:7

7. Halstead J, Redmond AC. Weight-bearing passive dorsiflexion of the hallux in standing is not related to hallux dorsiflexion during walking. J Orthop Sports Phys Ther. 2006:36:550-6.

8. Jack EA. Naviculo-cuneiform fusion in the treatment of flat foot. J Bone Joint Surg Br. 1953;35:75-82.

9. Munteanu SE, Landorf KB, McClelland JA, Roddy E, Cicuttini FM, Shiell A, Auhl M, Allan JJ, Buldt AK, Menz HB. Shoe-stiffening inserts for first metatarsophalangeal joint osteoarthritis (the SIMPLE trial): study protocol for a randomised controlled trial. Trials. 2017;18:198.

10. Redmond AC, Crosbie J, Ouvrier RA. Development and validation of a novel rating system for scoring standing foot posture: the foot posture index. Clin Biomech. 2006;21:89-98.

11. Zammit GV, Munteanu SE, Menz HB. Development of a diagnostic rule for identifying radiographic osteoarthritis in people with first metatarsophalangeal joint pain. Osteoarthritis Cartilage. 2011;19:939-45. 
12. Hopson MM, McPoil TG, Cornwall MW. Motion of the first metatarsophalangeal joint: reliability and validity of four measurement techniques. J Am Podiatr Med Assoc. 1995;85:198-204.

13. Menz HB, Munteanu SE, Landorf KB, Zammit GV, Cicuttini FM. Radiographic classification of osteoarthritis in commonly affected joints of the foot. Osteoarthritis Cartilage. 2007;15:1333-8.

14. Nester CJ, Jarvis HL, Jones RK, Bowden PD, Liu A. Movement of the human foot in 100 pain free individuals aged 18-45: implications for understanding normal foot function. J Foot Ankle Res. 2014;7:51.

15. Kadaba MP, Ramakrishnan HK, Wootten ME, Gainey J, Gorton G, Cochran GV. Repeatability of kinematic, kinetic, and electromyographic data in normal adult gait. J Orthop Res. 1989;7:849-60

16. Davis RB, Õunpuu S, Tyburski D, Gage JR. A gait analysis data collection and reduction technique. Hum Mov Sci. 1991;10:575-87.

17. Cohen J. Statistical power analysis for the behavioral sciences. 2nd ed. Hillsdale, NJ: Erlbaum; 1988

18. Zammit GV, Menz HB, Munteanu SE, Landorf KB. Plantar pressure distribution in older people with osteoarthritis of the first metatarsophalangeal joint (hallux limitus/rigidus). J Orthop Res. 2008;26: 1665-9.

19. DeFrino PF, Brodsky JW, Pollo FE, Crenshaw SJ, Beischer AD. First metatarsophalangeal arthrodesis: a clinical, pedobarographic and gait analysis study. Foot Ankle Int. 2002;23:496-502.

20. Bojsen-Moller F. Anatomy of the forefoot, normal and pathologic. Clin Orthop Relat Res. 1979;142:10-8.

\section{Publisher's Note}

Springer Nature remains neutral with regard to jurisdictional claims in published maps and institutional affiliations.

Ready to submit your research? Choose BMC and benefit from:

- fast, convenient online submission

- thorough peer review by experienced researchers in your field

- rapid publication on acceptance

- support for research data, including large and complex data types

- gold Open Access which fosters wider collaboration and increased citations

- maximum visibility for your research: over $100 \mathrm{M}$ website views per year

At BMC, research is always in progress.

Learn more biomedcentral.com/submissions 